\title{
ON THE OPTIMAL MODES OF CONTROLLED TRANSFER OF WALKING PROPULSION DEVICES
}

\author{
M. V. MIROSHKINA and E. S. BRISKIN \\ Department of Theoretical Mechanics, Volgograd State Technical University, Lenin Avenue, 28, \\ Volgograd region, Russia \\ E-mail:dtm@vstu.ru,mariatiminen@yandex.ru \\ www.vstu.ru
}

\begin{abstract}
The problem of walking machine leg transfer is considered. Optimal laws of transfer are determined with regards to geometrical features of ground underwater. Complex optimality criterion is introduced as sum of indexes of quality of the movement multiplied each by weight coefficients. The solution is presented based on walking machine "Ortonog".
\end{abstract}

\section{Introduction}

Mobile robots with walking propulsion devices are capable of overcoming obstacles without any physical contact with them and have prerequisite to obtain perfect maneuverability. The perfect maneuverability means that body of robot can make any predefined plane motion [1]. This is the main advantage of walking mobile robots in comparison with traditional types of drives (wheels, trucks, etc.). The disadvantages are low speed and energy efficiency. This is caused by the properties of the walking machines as an unbalanced mechanism to interact discretely with supporting ground in the process of transfer and ensure the movement of the foot along an arbitrary path [2].

In case of moving on unknown terrain there is still the need to ensure the movement of mobile robot without gliding or stumbling. The law of control to obtain this comfortability is known [3]. The knowledge of geometrical characteristics of ground (such as the height of obstacles, distances between them and to them etc.) can affect both law of control for leg transfer and its trajectory. For example, it can copy the ground profile.

The trajectory depends on optimality criterion. The latter can consist of several indexes, for example, minimum heat loss in drive motors, minimum rms power developed by engines, minimum distance traveled, etc $[4,5]$. These indexes can be compiled in complex optimality criterion to determine the boundaries of the Pareto-optimal motion modes [6].

For walking robots with orthogonal-rotary propulsion devices, for example, for the "Ortonog" machine (Figure 1), the law of vertical movement of the propeller foot is established in accordance with the optimality criterion consisting of several indicators [7] and separately horizontal [8].

However, the task of determining the optimal programmed foot movement of the propulsion devices of an underwater mobile robot both in the horizontal and vertical directions simultaneously while overcoming obstacles was not considered. This task is especially relevant for underwater robots, since movement is characterized by much greater forces of resistance to the movement of the feet in a denser compared to air environment, and the unevenness of the bottom, which makes it necessary to step over fairly large obstacles. 


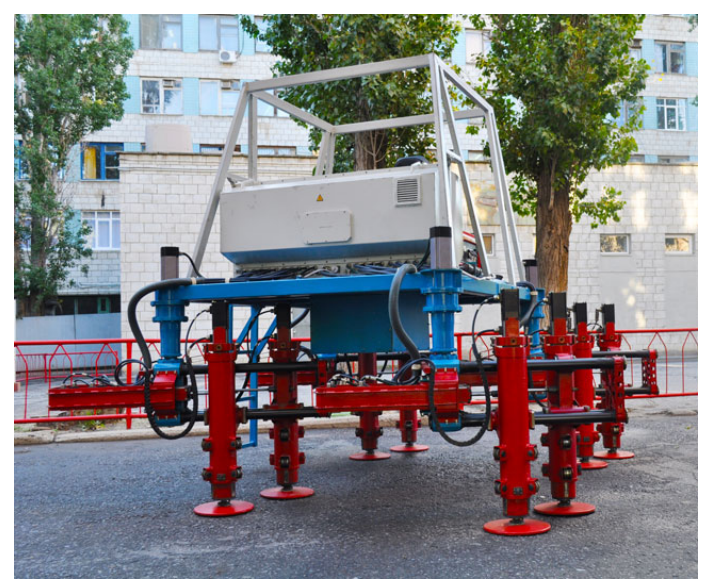

Figure 1. Walking machine 'Ortonog”.

\section{Statement of the problem}

The foot of underwater walking mobile robot is viewed as material point. The movement of the point in vertical plane is analyzed. In this case, the material point means the center of the foot of a walking robot, the optimal trajectory of which is determined in this paper. One transfer is characterized by following features: starting point $\mathrm{A}$, the length of step $\mathrm{L}$, the height of obstacle $\mathrm{H}$, the distance to the obstacle $\mathrm{S}$, destination point $\mathrm{B}$, the height of destination point $\mathrm{h}$ (Figure 2). Forces considering are: $\mathrm{P}$ - weight, forces of resistance to movement $R_{x}, R_{y}$, proportional to speeds of movement $V_{x}=\dot{x}, V_{y}=\dot{y}$, forces developed by drives of vertical $T$ and horizontal $F$ displacement.

The movement is described by the system of differential equations

$$
\left\{\begin{array}{c}
m \ddot{x}=F-\mu_{x} \dot{x} \\
m \ddot{y}=T-P-\mu_{y} \dot{y}
\end{array}\right.
$$

where $\mu_{x}, \mu_{y}$ - coefficients of viscous resistance of a liquid, depending on the shape and geometrical dimensions of the foot mass $m$.

Boundary conditions are set:

$$
\begin{gathered}
\text { at } t=0, x_{0}=0, y_{0}=0, \dot{x}_{0}=0, \dot{y}_{0}=0 ; \\
\text { at } t=\tau, x_{\tau}=L, y_{\tau}=h, \dot{x}_{\tau}=0, \dot{y}_{\tau}=0 .
\end{gathered}
$$

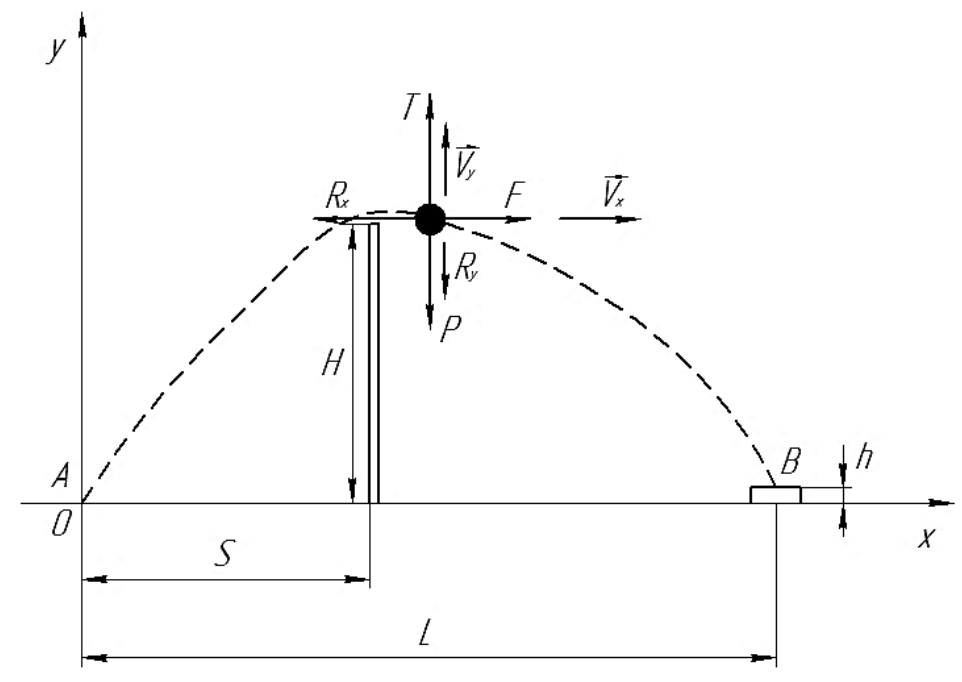


The dimensionless indexes of quality of the movement $I_{j}$ are introduced. The sum of these indexes form complex optimality criterion $I$ :

$$
I=\sum k_{j} I_{j}
$$

where $k_{j}$ - weight coefficients, defined up to a constant factor and selected in accordance with the significance of each index.

$$
I_{1}=\frac{1}{A}\left(\alpha_{1} \int_{0}^{\tau} F^{2} d t+\alpha_{2} \int_{0}^{\tau} T^{2} d t\right)
$$

where $I_{1}$ - index of quality of the movement, characterizing heat loss in drive motors, $\alpha_{1}$, $\alpha_{2}$ - known constants characterizing drive motors, $A$ - arbitrary reference work defined, for example, $A=m g H$.

$$
I_{2}=\frac{1}{\tau g^{2}} \int_{0}^{\tau} \ddot{x}^{2} d t ; I_{3}=\frac{1}{\tau g^{2}} \int_{0}^{\tau} \ddot{y}^{2} d t
$$

$I_{2}, I_{3}$ - indexes of quality of the movement, characterizing the square of rms acceleration, $g$ - acceleration of gravity.

$$
I_{4}=\frac{\tau}{A^{2}} \int_{0}^{\tau}\left[(F \dot{x})^{2}+(T \dot{y})^{2}\right] d t
$$

$I_{4}$ - index of quality of movement, characterizing the square of rms of power of the drives.

The task is to find the law of movement $x=x(t)$ and $y=y(t)$ to ensure the overcoming of obstacle by foot of walking machine, to satisfy the boundary conditions (2) and delivers minimum to at list one of the indexes (4) - (6).

\section{The method of solving}

The method of solving the problem is based on splitting the movement into two stages: before and after overcoming the obstacle.

First stage: $0<t<\tau_{1}$ :

$$
\begin{array}{cccc}
x(0)=0, & \dot{x}(0)=0, & y(0)=0, & \dot{y}(0)=0, \\
x\left(\tau_{1}\right)=S, & \dot{x}\left(\tau_{1}\right)=U, & y\left(\tau_{1}\right)=H, & \dot{y}\left(\tau_{1}\right)=0 .
\end{array}
$$

Second stage: $0<t<\tau-\tau_{1}$ :

$$
\begin{gathered}
x(0)=S, \quad \dot{x}(0)=U, \quad y(0)=H, \quad \dot{y}(0)=0, \\
x\left(\tau-\tau_{1}\right)=L-S, \quad \dot{x}\left(\tau-\tau_{1}\right)=0, \quad y\left(\tau-\tau_{1}\right)=h, \quad \dot{y}\left(\tau-\tau_{1}\right)=0 .
\end{gathered}
$$

Each stage is characterized by control parameters $\tau_{1}, U$. For each stage the problem of minimum of functional (3) must be solved with the help of Euler-Poisson equations [9]

$$
\begin{gathered}
\frac{d^{2}}{d t^{2}}\left(\frac{\partial \Phi}{\partial \ddot{x}}\right)-\frac{d}{d t}\left(\frac{\partial \Phi}{\partial \dot{x}}\right)+\frac{\partial \Phi}{\partial x}=0 \\
\frac{d^{2}}{d t^{2}}\left(\frac{\partial \Phi}{\partial \ddot{y}}\right)-\frac{d}{d t}\left(\frac{\partial \Phi}{\partial \dot{y}}\right)+\frac{\partial \Phi}{\partial y}=0 \\
\Phi=\frac{k_{1}}{A}\left[\alpha_{1}\left(m \ddot{x}+\mu_{x} \dot{x}\right)^{2}+\alpha_{2}\left(m \ddot{y}+P+\mu_{y} \dot{y}\right)^{2}\right]+\frac{k_{2}}{\tau g^{2}} \ddot{x}^{2}+\frac{k_{3}}{\tau g^{2}} \ddot{y}^{2}+ \\
+\frac{k_{4} \tau}{A^{2}}\left[\left(m \ddot{x}+\mu_{x} \dot{x}\right)^{2} \dot{x}^{2}+\left(m \ddot{y}+P+\mu_{y} \dot{y}\right)^{2} \dot{y}^{2}\right] .
\end{gathered}
$$


If $k_{4}=0$ then equations (9) become

$$
\left\{\begin{array}{l}
x^{\mathrm{IV}}-\lambda_{x}{ }^{2} \ddot{x}=0 \\
y^{\mathrm{IV}}-\lambda_{y}{ }^{2} \ddot{y}=0
\end{array}\right.
$$

where

$$
\lambda_{x}^{2}=\frac{\frac{\alpha_{1} k_{1} \mu_{x}^{2}}{A}}{\frac{\alpha_{1} k_{1} m^{2}}{A}+\frac{k_{2}}{\tau g^{2}}} ; \lambda_{y}^{2}=\frac{\frac{\alpha_{2} k_{1} \mu_{y}{ }^{2}}{A}}{\frac{\alpha_{2} k_{1} m^{2}}{A}+\frac{k_{3}}{\tau g^{2}}} .
$$

Equations (10) are solved at each stage with regards to boundary conditions (7), (8).

The optimality of foot transfer according to selected criterion (3) at each stage together with the choice of control parameters $\tau_{1}, U$ provide the optimality of entire movement mode [4].

The solutions of differential equations (10) at each stage are having following form

$$
\begin{gathered}
x(t)=C_{1}+C_{2} t+C_{3} e^{\lambda_{x} t}+C_{4} e^{-\lambda_{x} t} \\
y(t)=B_{1}+B_{2} t+B_{3} e^{\lambda_{y} t}+B_{4} e^{-\lambda_{y} t}
\end{gathered}
$$

Constants $C_{i}, B_{i}(i=1 \ldots 4)$ are determined from the boundary conditions.

As a result, all indexes of quality of movement and complex optimality criterion are determined in accordance with differential equation of transfer movement (1) and expression (11).

$$
I=\int_{0}^{\tau} \Phi d t
$$

\section{The model task of determining the optimal mode of movement and analysis of results.}

In order to solve the problem, it is initially required to determine the control parameters $\tau_{1}, U$ that provide minimum for complex criterion (12), or one of indexes of quality $I_{j}$, for example, index of heat loss $I_{1}$.

For model task let's consider the walking mobile robot "Ortonog" with dual walking mechanism. The body of robot is moving uniformly rectilinearly at a constant speed $V$. The characteristics of mechanism are: the mass $m=70 \mathrm{~kg}$, the stride length $L=0,91 \mathrm{~m}$, constants characterizing drive motors $\alpha_{1}=\alpha_{2}$. The speed $V$ is related to the stride length and the foot transfer time $\tau$ as

$$
V=\frac{L}{2 \tau}
$$

The basic ground profile and robot position are characterized by the following parameters $H=0,3 \mathrm{~m}, S=0,3 L=0,273 \mathrm{~m}, h=0$.

Thus, the variable parameters are the constructive $\lambda_{x}, \lambda_{y}$, which characterize the streamlining of the propulsion device by the external environment - water, the speed of the robot $V$, the desired control actions $U=\gamma V, \tau_{1}=\varepsilon \tau=\varepsilon(L / 2 \tau)$, which are convenient to specify dimensionless parameters $\gamma$ and $\varepsilon$, and the geometric characteristics of the soil profile $H, S, h$, varying depending on specific conditions.

The mathematical modelling shows the dependence of indexes of quality (4), (5) included in complex criterion (3) from control parameters.

On the Figure 3 the dependence of index of heat loss in drive motors $I_{1}$ on dimensionless control parameters $\varepsilon=\tau_{1} / \tau$ and $\gamma=U / V$ is shown for known geometric characteristics of the 
ground profile and various values of the vehicle speed. The graphs show that there is an optimal mode of foot transfer over an obstacle, characterized by the parameters of the machine. Their analysis shows that at low and high values of the speeds of movement of a mobile robot, the index of heat loss $I_{1}$ reaches high values, while at a certain value of $\mathrm{V}$ the index $I_{1}$ reaches a minimum.
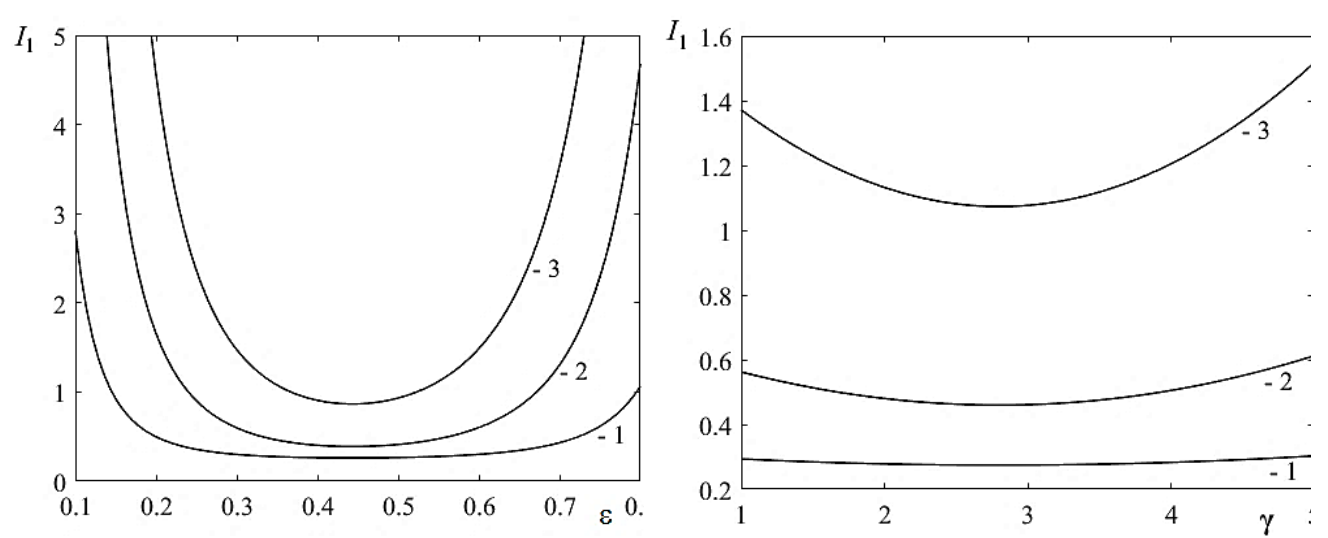

Figure 3. The dependence of index of heat loss in drive motors $I_{1}$ on dimensionless control parameters $\varepsilon$ and $\gamma$ : $1-V=0,4 \mathrm{~m} / \mathrm{s} ; 2-V=0,7 \mathrm{~m} / \mathrm{s} ; 3-V=1 \mathrm{~m} / \mathrm{s}$.

Dependences of the index of heat loss in drive motors $I_{1}$ (Figure 4) and the dimensionless square of the rms horizontal acceleration $I_{2}$ (Figure 5) on the dimensionless $\varepsilon=\tau_{1} / \tau$ and $\gamma=U / V$ for various geometric parameters of the ground profile, characterized by the location of the obstacle S. Their analysis shows that there is an optimal mode of foot transfer over an obstacle.
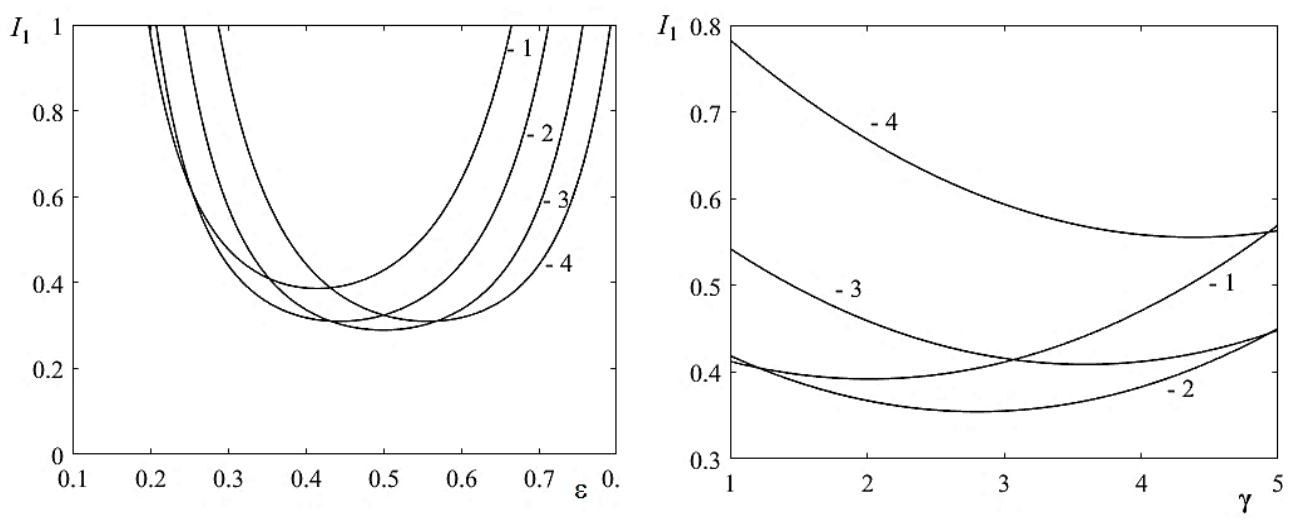

Figure 4. The dependence of index of heat loss in drive motors $I_{1}$ on dimensionless control parameters $\varepsilon$ and $\gamma$ : $1-S=0,1 L ; 2-S=0,3 L ; 3-S=0,5 L ; 4-S=0,7 L$ 

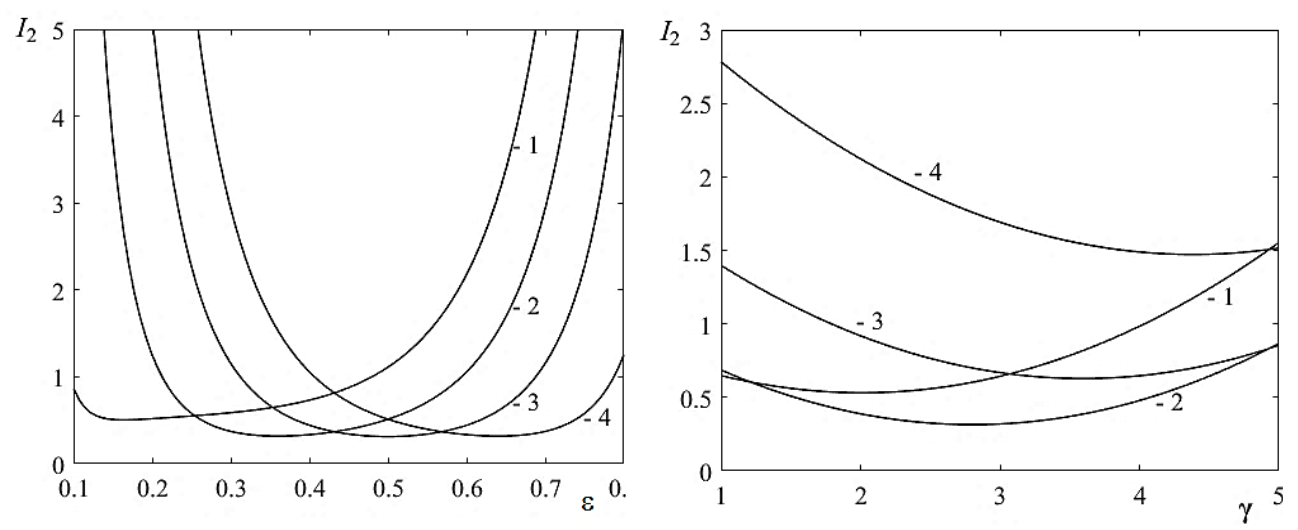

Figure 5. The dependence of the index of the dimensionless square of the rms horizontal acceleration $I_{2}$ on the dimensionless $\varepsilon$ and $\gamma$ :

$$
1-S=0,1 L ; 2-S=0,3 L ; 3-S=0,5 L ; 4-S=0,7 L \text {. }
$$

The optimal modes of movement shown in the figures (3 - 5) consider only one of the possible indexes. If we consider the significance of each of the indexes by weight coefficients $k_{j}$, then the particular indexes change (Figures 6,7 ) and are controversial. With the growing importance of one of them, the other index decreases. The analysis of such dependencies allows us to determine the Pareto optimal boundaries, which is decision support for the robot control system.
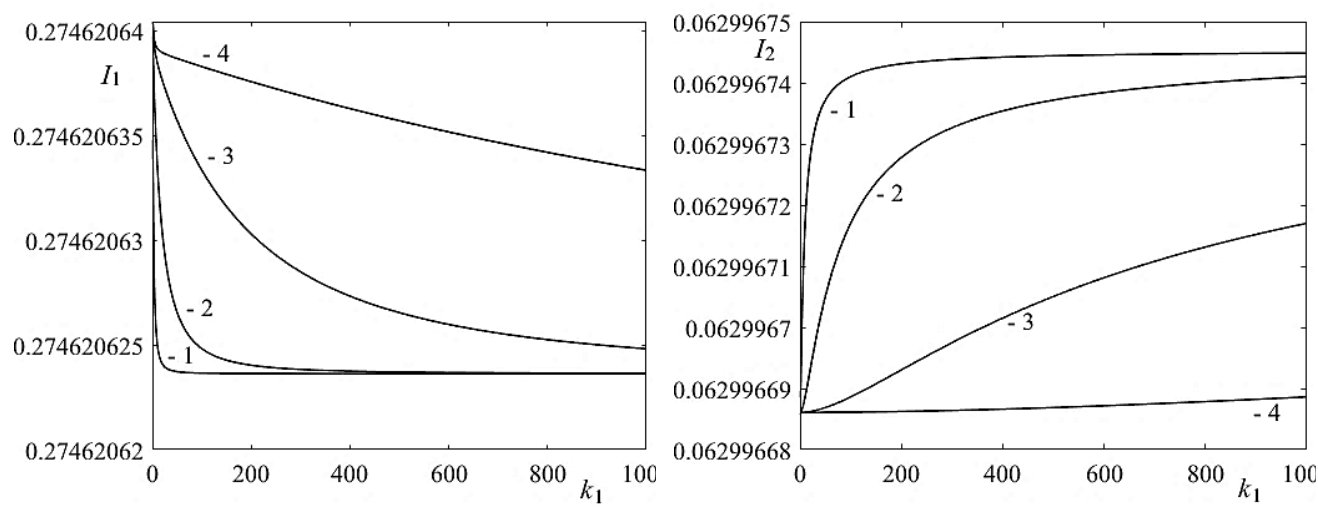

Figure 6. Dependences of the index of heat loss in drive motors $I_{1}$ and the dimensionless square of the rms horizontal acceleration $I_{2}$ on weight coefficients $k_{1}$ :

$1-k_{2}=1 ; 2-k_{2}=10 ; 3-k_{2}=100 ; 4-k_{2}=1000$. 

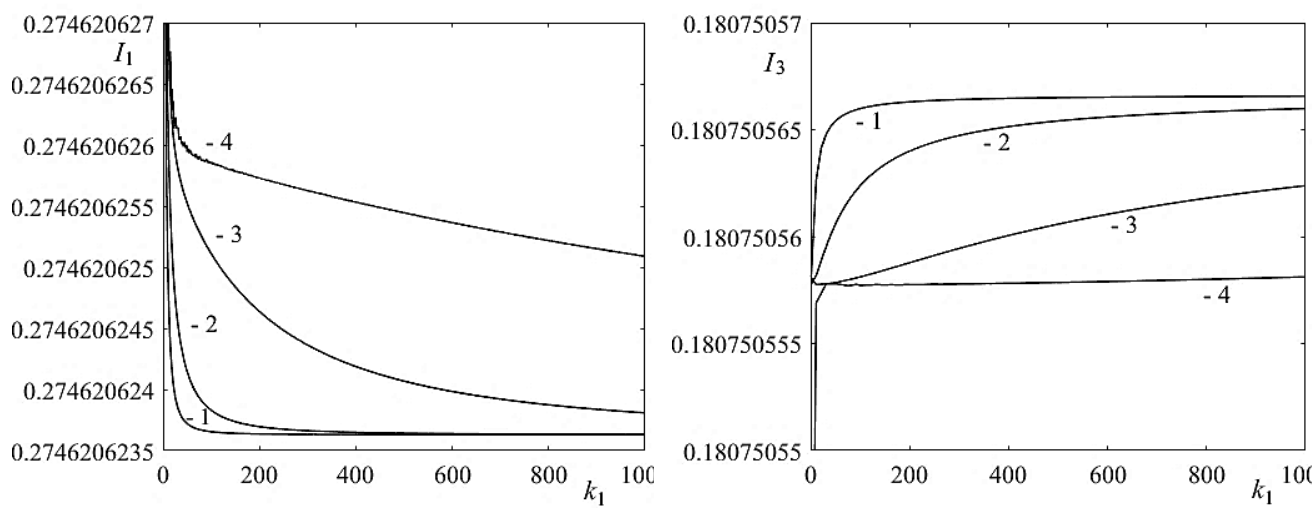

Figure 7. Dependences of the index of heat loss in drive motors $I_{1}$ and the dimensionless square of the rms vertical acceleration $I_{3}$ on weight coefficients $k_{1}$ :

$$
1-k_{2}=1 ; 2-k_{2}=10 ; 3-k_{2}=100 ; 4-k_{2}=1000 \text {. }
$$

\section{Conclusions}

A design scheme is proposed and a mathematical model is developed to describe the dynamics of the movement of the transfered foot of the walking propulsion device of an underwater mobile robot.

Indexes of quality of movement are formulated that determine the optimal control modes for the transfer of the foot of a walking propulsion device when overcoming obstacles, and methods for their determination are developed.

The dependences of indexes of quality on the speed of the robot, the control parameters $\varepsilon$, $\gamma$ and the geometric parameters of the ground profile $S, H$ are determined.

A method for constructing the Pareto-optimal boundaries of variable parameters has been developed.

The proposed solution of the problem poses a significant advantage along with the already known approaches to solving such problems through the use of a developed informationmeasuring system and the combination of the simultaneous movement of orthogonal devices in the horizontal and vertical directions.

The calculated data and dependencies are planned to be confirmed experimentally.

\section{Acknowledgments}

The reported study was funded by RFBR, project number 19-31-90112.

\section{References}

1. Briskin, I.P. Vershinina, A.V. Maloletov, N.G. Sharonov, Journal of Computer and Systems Sciences International 53, DOI: 10.1134/S1064230714020038 (2014).

2. D.E. Okhotsimsky, Yu.F. Golubev, Mechanics and motion control of an automatic walking apparatus (1984).

3. A.P Bessonov, N.V. Umnov, V.V. Korenovsky et al, Six Link Mechanisms for the Legs of Walking Machines, Romansy 13, Theory and Practice of Robots and Manipulators, DOI: https://doi.org/10.1007/978-3-7091-2498-7_37 (2000).

4. E.S. Briskin, Y.V. Kalinin, M.V. Miroshkina, Bulletin of the Russian Academy of Sciences. Theory and control systems DOI: 10.31857/S0002338820010035 (2020).

5. E.S. Briskin, Y.V. Kalinin, A.V. Maloletov, V.V. Chernyshev, Mechanics of Solids 49, DOI: $10.3103 / \mathrm{S} 0025654414010026$ (2014). 
6. E.S. Briskin, Y.V. Kalinin, A.V. Maloletov, V.A. Shurygin, Journal of Computer and Systems Sciences International 56, DOI: 10.1134/S1064230717020058 (2017).

7. E.S. Briskin, Y.V. Kalinin, A.V. Maloletov, V.A. Serov, S.A. Ustinov, Journal of Computer and Systems Sciences International 56, DOI: 10.1134/S1064230717030078 (2017).

8. E.S. Briskin, Y.V. Kalinin, Journal of Computer and Systems Sciences International 50, DOI: 10.1134/S1064230711020043 (2011).

9. E.L. Elsgolts, Calculus of variations (1961). 